\title{
[ 4251
}

IV. Unterfuchungen über eine befondere Eigenfchafo der metallifchen Elektricitätsleiter; vom Hrn. Prof. August de la Rive.

(Gelefen in der Societe de physique et d'histoire naturelle zu Genf, am 22. Jun. 1826. Aus der Bibllothèque universello T. XXXV. p. 92.)

Am Schlnthe einer früheren Abliandlung habe ich gefagt, dafs die metallifchen Leiter der Voltafchen Elaktricital, welche zur Zerfetzung einer Flüfigkeit benutzt worden find, noch aufserhalb der Kette eine gewifle Menge von Elektricitast behalten, und dafs Ge, unter günftigen Umftünden, einen Strom von bemerkenswerther Intenfität hervorbringen können "). Es

$\Rightarrow$ Annal. de chim. et de phys. XXVILl. 190. - Diefe Abhandlung, fo wlo die verwandien Arbeiten des Hrn. Marianini, folien den Lefern, wenn auch nur auszugsweife, in einem der nächflen Hefte mitgetheilt werden. Vorlaufig finde bier der Schlufs diefer Abhandlung eine Stelle, da der mit dem Texte in năchner Beziehung Ateht. "Ritter, in feiner Arbeit Wher dio Secundăren Sżulen (Journal do physique T. LVIl. p. 3450 . 367), hatte bemerkt, dafs eine Reihe metallifcher Platten votr gleicher Natur, die, in gleichen oder ungleichen Abwechslungen, durcb fouchto Pappicheiben unterbrochen, und in den Kreis der Säule gebracbt worden war, eine merkliche Elektricitat behielt, doch nur auf kurze Zelt. In den, dea fecuadiren Säulen ähnlichen, Apparaten, deren ich mich bediente, um den Strom durch eine ron Metallplatten unterbrochene Fluiffigkeit zu leiten, fah ich, dafs auch nach Aufhebung der Rette nocb Elektricitat auftrat. Ich tberzeugte mich, dafs fie von dell Metallplatten, and keineswieges ron der Flungigkeit herrührte; und es fehien, dafs as die von den einzeluen Platten AunaL. d. PhyGk. B. 86. St.3. J. 2827. St. 7.

E。 


\section{[ 4:6 ]}

Jhat mir gefchienen, dafs diefe Eigenfchaft ein Corgfaltigeres Studium verdiene, und es find einige hierüber

whhrend threr Anwefenheit in dem Kreifo der Säule zuriick. gehalterze Elektricitat war, welche Iich fpăterhin zeigte, wenn der Strom unterbrochen wurde. Die Platinleiter, welche die Pole mit der 24 zerfetzenden Fluffigkeit verbinden, behalten eine ziemlich beträchtliche Menge von Elektricitat, wie es der Galvanometer anzeigt. Um fich davon zu iberzeugen, braucht man fie nur, nachdem fie eive Weile als Pole der Szule gedient haben, fortzunehmen und an dit Enden eines Galvanometers zu bringen. So wie diefs gefchieht und man fie in einen und denfelben fluffigen Leiter taucht, telle fich ein Strom ein von folcher Richtung, dafs er dea Metalldraht des Galvanometers durchliuft von dem Drahte aus, der als + Pol gedient hat, zu demjoniget, dor als - Pol gedient bat.

"Schon vinet der Drăbte, die als Pole gedient habet, reicht hin, oinen Strom zu bewirken, wenn nur das andero Ende des Galvanometers fict in einem Platindrabte endige, der mit dem frühet als Pol gebrauchton in diefelbe Flomgkeit taucht. Die Richtung des Stroms bängt davon ab, mit welchem Polo der Saule der angewandte Platindrabt zuror verbunden gewefen ift. Es if merkwürdig, dafs diefe Drahte ziemlich lango thre Eigenfchaft behalten, einen Strom zu er. regen, und dals weder Berührung noch Reibung diefelbe zerftärez kano, Sollte diefe Elektricität eino Spannungss elektricitat feyn, wio fie von den Elektrifirmalchinen eiregt wird? Wias ich von den an den Enden der Szulo befellige gewefenen Leitdralten gefagt habes gilt in glelcher Weife fär jeden mes tallitchen Leiter, welcher in der Flüligkeit rom elektrifchen Strome getroffen wird. Daher zeigt der Apparat, wonn er nicht nata im Kreise der Săule ift, und wenn man feine Enden durch den Draht des Galvanometers verbindet, einen elektrifchen Stroun. Ich bemerko noch, dars die Vertheilang der Elektricităt in dea leitenden Platten mir kein gleichformiges and conftantes Gefetz 20 befolgen fchien, weder was 


\section{[427]}

erhaltene Refultate, wolche ich die Eliro habo der Gofellchliaft mitzuthoilen.

die Intenfität, noch was die Richtung des Stroms betrifft. Sie weicht in diefer Bezielung ron der Vertheilung $2 b$, welche Ritter mittelf chemifcher Zerfetzungen und phyfialogifcher Wirkungen beobachtot bat, oiner Methode, dio mir weniger empfindlich und genau fcheint als die, welche aus dem Gebrauche des Galvanometers hervorgeht." -

Man fieht fchon hieraus und kann fich noch mehr durch Lefung jener altern Abhandlung oder der Schriften von Rittor uberzeugen, dafs die tm Texte von Hro. Do la Riro befchriebene Ericheinung im Grunde nicht unbekannt gewefen ift. Ritter, wie namentlich Oersted in der orwahnten Abhandlung, won Journ. de pliysique, bemerkt, ift durch fio auf die Confruction der Ladungsfiule gefliht worden. Indefs wird die Mitheilung der Arboit des Hra. De la Rive nicht uberfurfig feyn, da fio die Haupterfcheinung durch die damals noch unbekannte magnetilche Wirkung der Kette betäfifr, manches Neue enthait, und endlich die ron $\nabla$ olta aufgoftellio and vielleicht noch von Einigen gehegte Meinung, als sey die Ladungsfule nur eine Siule aus einem metallifichen und zwei flufigen Leitern, wohl vollends widerlege Es verdient auch noch bemerkt 24 werded, dals die neuerlich von Hrn. De I a Ri ve (in feiner ron thm felbst citirten Abhabdlong) ind Hrn. Marianin i beobachtete Abrabme der Leitungsfaligkeit einer Flafigkeit durch Unterbrechung mit Metallplatte.s, fchon von Ritter wahrgenommen Ift, und diefem, wie jenem, Gelegenheit gegeben hat, auf die Aehnlichkeit in der Fortplan. zung der Elektricitat und das Lichtes aufmerkfam zu machen. Ritter fongt noch, als eino $z$ weite Aehnlichkeit diefer Arf, hinzu, dais die Wirkung der Sxule fich in gerader Lifoie fortpflanze, wenigftens, dafs ein Eifeadraht bedeutend an Leitungsfohigkeit vorliere, wena man lbn krlimm oder in ein Zickzack biege. Oersted verfichert, den Verfach relb? ron Ritser gefeluen zu haben; or lf daher gewifs eloer naheren 


\section{$\left[\begin{array}{lll}428 & \end{array}\right]$}

Grundthatfache. Ich Jalfo dio beiden Pole einer Saule in zwei Platindrăhten endigen, und tauche diefo in eine Löfung von Salmiak oder irgend einem andern, durch fie zerfetzbaren flüfligen Leitcr. Nachdem die Zerfetzung eine Weilo gedauert liat, nelıme ich die Druhte fort, and bringe fie mit den Enden eines Galvanometers in Verbindung, wahrend ein fliiffiger Leiter diejenigen Theile dieler Drähte aufnimnit, welche zuvor in die, in den Kreis der Suule gebrachte, Flüfligkeit eingetaucht waren. Soglciclı zeigt die Ablenkung der Nadel das Dafeyn eines elektrifchen Strome im Galvanometer an, und die Art der Ablenkung beweift, dafs der Strom, von dem ich annelıme, er gehe von den zuvor am pofitiven Pole der Säule befindlichen Drahte aus, zunăchit den Metalldraht des Galvanometers durchluuft, dadurch zum andern Platindraht, der els negativer Pol der Saule diente, gelangt, und von hieraus durch die Flülfigkeit zu dem Drahte, von dom wir ausgegangen find, übergeht, wodurch dann die Ketto gefchlolen ift. Mit andern Worten: Derjenige Draht,, welcher an der Süule als pofitiver $\mathrm{Pol}$ gedient hat, wirkt wie ein negatives $\mathrm{Me}$ tall, und der, welcher als negativer Pol benutzt worden ift, wie ein pofitives Metall, wenn fie beide an die

Berückfichtigang werth, 2umal die neueren Unterfuchungen iho ganz abergangen haben. Mit der von Hrn. De la Rive befcbriebenen Erfcheinung fcheint lubrigens die von Hrn. Arago gemachte und im vorigen Bande S. 443 angefiuhrte Beobachtung, dafs Platindralute, die aus dem Kreife der Saulo genommen werden, zuweilen noch Eifeufeile anziehen, in unmltzelbarer Bezielung zu ftelien.

P. 


\section{[ 439 ]}

Enden des Oalvanometers gebracht werden. Es folgt darans, dafs der Strom in der Flülfigkeit, welche diefe beiden Enden verbindet, in Bezug auf die beiden Platindrälte, gerado in umgekehrter Richtung gelit, wio der, welcher unter dem Einflufie dor Săule die FlüfSigkeit durchliunft"\%. Linzu:

Icl füge diefem noch folgende Beobachtungen

1) Der von den beiden Platiodrahten erregte Strom ift nicht blols augenblickliclı. Er dauert eine Weile und man kann die Drähte felbft mehrmals eintauchen, ehe fio vollftindig entladen werden.

2) Es ift zum Gelingen des Verfuchs nicht nôthig, dafs man gerade dasjenige Stück der beiden Platindrähte, welclies zur Zerfetzung gedient hat, eintauclıe; man kann das Stück des Drahts, welclies frch in dem Voliafchen Strome aufserhalb der Flïfligkeit befand, zerfchneiden und eintauchen; die Wirkung ift nur fchwscher, und um fo mehn, als der eingetauchte Theil fich weiter von dem entfernt, an welchem die Zerfetzung vor fich ging. Diefe Bcobachtung beweift, dafs es nicht eine chemifclid Wirkung der leitenden Flärfigkuit auf das Element der zerfetzten und möglicherweile am Platindralıte lıängon geblicbenen Salzlöfung fey, von welchicr die Erfchicinung herrülirt; fondern, dufs es eine belondere Eigenfchaft der Metalldrälıte ift, die der Voltafehen Elektricitat als Leiter

*) Ich werde mich nicht mit einer ausfuhrlichen Bofchreibung der Art, wie ich diefe Vcrfuche gemaclit habe, auflialten; fondern bemerke nur, dals alle Verbindungen mittelit Queckalbers bewerkflelligt worden find. 


\section{[ 430 ]}

gedient haben; eine Eigenfchaft, die durchiaus von der gew 8 huliohen Elektricitat verfchieden ift und die jch daher, um fie von diefer zu unterfcheiden, elektrom dynamifche Eigenlchaft nennen werde. Icls habe mich übrigens direct überzangt, dafg bei der Erzeugung divler Erfolieinung nichts vorhanden ift, das von einer chemifchen Wirkung des flülfigen Leiters herrührte, weder an den Platindraliten, noch an dem Antheile der zerfetzten Flülfigkeit, dio an diefen Druliten hängen geblieben wđre "\%.

3) Ein cinziger Draht reicht hin, den Strom zu beftimmen, vorallggefetzt, dafs das andere Endo des Galvanometers mit der Flüligkeit in Gemeinfcliaft ftelıe, in welche diefer Dralit getaucht ift. Der Leiter von Platin, welcher zur Herftellung diefer Communication dient, vertritt alfo die Stolle eines negativen Metalles, wenn der am Galvanometer befeftigte Dralıt derjenige ift, welcher am negativen Pole der Süule befindlich gevefen ift, und die eines poftiven Metalles, wenn er am pofitiven Pole gewefen ift. Die Wirkung ift dann immer geringer (lialb lo grofs) unter denlelben L'mftünden, wie mit zwei slektrifirten Drähten.

Zwei wiehtige Umfiznde fcheinen von Einflufs zu feyn auf die Intenfi:at des Stroms, der eine, die Zeit, walhrend welcher die beiden Dralhte der Wirkung der Siule unterworfen gewefen find, der

7) Ich überzeugte mlch immor forgfallig, dafs dio angewandten Platindrahto fur fich keinon Strom erregten, wann fie, an den Enden des Galvanometers befelligt, in den flügligen Leiter getaucbt wurder. 


\section{[ 43x]}

andere, die Natur der Loiter, welcho den Bogen fchliefsen.

I. Die Zeit. Als ich zum erfen Male den früher nur zufälig gemachiten Grundverfuch wiederholte, überral'clite es mich felır, dafs er nicht gelang. Icls fah aber bald ein, dafs diefs daher rührte, dafs ich die Drähte nur einige Augeriblicke in den Voltafchen Strom gelaten hatte; als ich fie langere Zeit darin liels, erhielt ich nerkliche Wirkungen, und um fo betrachtlichere, je langer die Zeit war. So erhielt ich, als alle übrigen Umftande diefelben blieben, linfichtlich der Zeit

$$
\text { Nach: } \begin{array}{ccccc}
1 & 2 & 3 & 4 & 5 \text { Minuten } \\
60^{\circ} & 65^{\circ} & 70^{\circ} & 75^{\circ} \text { bis } 80^{\circ} & 85^{\circ} \text { Ablenkung }
\end{array}
$$

Der Einflufo der Zeit machte fich, felbft über einige Minulen hinaus, noch merklich, aber weniger in Betreff der Intenfitat des Stroms, als unter zwei andern Gelichtspunkten.

1) Jo langer dic Dralite der Wirkung der Voltafchen Säulen ausgefelzt waren, defto lünger behielten fie die elektrodynamifche Eigenfchaft, welche Operation man anch mit ihnen vornelumen mochte. Ein Leildraht, der 20 bis jo Minuten dem Strome überlallen, und darauf gewal'clien und abgetrocknet worden ift, befitzt nocl nach Ablauf einiger Tage die Eigenfchaft, einen Strom zu orregen.

2) Die Dauer des Stroms, den man durch Iueiter erregt, welche eine malsig lange Zeit hindurch der Virkung der Saule ausgeletzt gewefen find, ift viel betrichtlicher, als der von den Drahten, auf welche die Suule nur einige Augenblicke gewirkt hat. 


\section{[ 432 ]}

Es gelit alfo aus dem Obigen hervor, dafs der Zuftand, in den die Dralute verfetzt werden, wenn fie als J.eiter der Voltafchen Elektricitat dienen, nicht attgenblicklich eintritt, fondern erft nach Verlauf einer mehr oder weniger langen Zeit. Ich habe aber kcinen Vortheil davon gefehen, wenn ich die Leiter langer als 30 Minuten in dem Strome liefs.

II. Natur der Leiter. In den vorlsergehenden Verfuchen habe ich nur zerfetzbare Flüfligkeiten angewandt, fowohl um die Drahte in den Krois zu bringen, als auch, um Go zu verbiniden, wenn fio an den Enden des Galvanometer befeftigt find. Mit den beffercn Leitern, d.h. mit den metallifchen Leitern, habo ich nie eine bemerkbare Wirkung erhalten.

Wenn ich z. B. die beiden an den Enden der Saule befelligten Metalldrahte durch Queckfilber oder unınittelbar mit einander verband, und fie nun an die Enden des Galvanometers brachte, erhielt ich keine Wirkung, ich mochte fie durch einen metallifchen oder durch einen flüffigen Leiter verbinden. Dennoch war der Strom, der in diefem Falle den Draht durchJanfen latte, weit kraftiger, wie es ein Galvanometer bewies, der fo geftellt war, dafs er inmer die Intenfitait des von der Săule erregten Stromes angeben mufste. Keinen befern Erfolg fah ich, als icl die an den Enden des Galvanometers befeftigten Drahte, die zulvor zt:r Zerletzung gedient hatten, durch einen metallifchen Leiter verband; und was fonderbar ift, fio hatten durch diefe Berührung nichts von ihrem elektrodynanifclien Vermügen verloren, denn man konnto es entwickeln, wenn man darauf als $Z_{\text {wilchenmittel }}$ eineu dülligen Leiter anwandte. 


\section{[ 435 ]}

Es fcheint demnach nöthig zu feyn, dals der Bogen einen unvollkommnen Leiter einfchliefso, gleich als wenn der Widerftand, den die Elektricitat beim Durcligange durch denfelben erleidet, oder d̀̀o Verringerung der Gofch windigkeit des Stromes, den Drälsten erlaubte, eine Portion diefer Elektricitat zurückzulialten oder vielmehr ilnen Zeit liefse, fich in den Zufland zu verfetzen, der fie fühig machte, die beobachtete Erfcheinung hervorzubringen.

So unumganglich es aber ift, dafs der Bogen einen flüfrigen Leiter einfchliefse, eben fo nöthig ift es, dafs diefer oin möglichlt guter Leiter fey. Mehrere Verfuche haben mir bewiefen, dafs, in derfelben Zeit, die Leiter eine um fo kraftigere elektro-dynamilche Wirkung erlangen, als der Leiter, fowohl der an der Săule, als der an dem Galvanometer, vollkommner ift. Die Drähte, welche mit reinem Waffer mehr als 15 Minuten lang in dem Kreife der Süule gelafen wurden, erzeugten niemals eine gröfsere Ablenkung als 10\%, felbft wenn fie am Galvanometer durch einen guten flüffigen Leiter verbunden waren, und eine noch fchwachere, wenn fie hier ebenfalls durch reines $W$ afler in Verbindung gefetzt wurden. Mit einer fohwachen Löfung von Salmiak erzengten fie eine Ablenkung von 40 bis 50 Gradell, mit einer ftärkeren, wenn fie nur eine Minute im Strome gelalfen wurden, eine Ablenkung von $60^{\circ}$, nach zwei Minuten eine von $65^{\circ}$ and $70^{\circ}$, und nach einer langeren Zeit eine von $9^{\circ}$. $\mathrm{V}$ enn die Leiter mit einer felır concentrirten Salmiaklöfung oder mit reiner Schwefelfunre nur eine Minute lang der Wirkung der Säule ausgefetzt werden, gebon fie $g^{\circ}$ Ablenkung, und nach zwei Minu- 


\section{[ 434 ]}

ten unǵefăhr $180^{\circ}$ (? P.). Boi diefen Verfuchen zeigte der Galvanometer der Saulo den relativen Grad des Leitvermögens der Flüffigkeit an, und es fand fich, dalo diefes ziemlich genau in Verhultnifo zum Grado der Intenfitat des an die Leiter übergegangenen elektrodynamifchen Vermögens ftelie. Ich bemerke beiläufig, dafs es mir fchien, als fey die Intenfitat diefes Vermögens umgekelırt proportional dem Leitvermögen des als Leiter gebraucliten Metalls.

Iclı habe fchion bemerkt, dafs alle diefo Verfucho diefelben Refultate geben, wenn man den Theil der Leitdrahte, welcher zur Zerfetzung gedient hat, wafcht oder reibt. Aber die Wirkung wird jedesmal in einem gewiften Verhaltnillo verringert, was, wie ich glaube, theils davon leerrührt, dafs alsdann die Berührung zwifchen dem Dralıte und der Flüfligkeit weniger vollkommen ift, theils davon, dafs die Stärke des elektrodynamifclien Vermögens vom Ende des Dralites ab, wo fie am farkften ift, abnimmt,

Es folgt aus dem Vorhergehenden, dals die Gegenwart eines zerfetzbaren flüffigen Leiters in der Ketto nothwendig ley, wenn der Leitdralit die elektrodynamifche Eigenfchaft erlaalten foll. Sollte diefe Erfcheinung nicht mit denjenigen in Zulammenhang ftehen, welche fich beim Durchgange des elektrilchen Stromes durch mit einander abwechfelnde metallifcho Leiter und Flüfligkeiten, darbieten; Erfcheinungen, die den Gegenftand der Abliandlung ausmaclien, die 2u Anfange der gegenwarligen erwahnt wurde? Offenbar licheint es aber zu feyn, dafs die Flüfligkoiten, als Leiter, eine ganz befondere Wirkung auf die elektrifchen Ströme ausüben, welche vielleicht entfernte, 


\section{[ 4335$]$}

obgleich deshalb nicht weniger wirkliche, Aehnlichkeit nit gewiflen Erfcheinungen des Lichts und der Warme darbietet.

Verfuch en einer Theorie. Vor Unternehmung einer neuen Reihe von Verfuchen iff es wohl nicht usnnitz, von den bereits beobachteten Erfcheinungen eine Erklarung zu geben zu fuchen, damit man wifle, nach welcher Seite liin die Arbeit mit Vortheile zu lenken fey.

Zwei Umftande fcheinen vor Allem wichtig und müfen bei Anffuchung einer Theorio leiten: 1) dio Richlung des von den Leitdrahten erzengten Stroms, und 2) die Nothwondigkeit eines flüffigen Leiters zum Auftreten der Erfclieinung. Die folgende Erklarung ift auf eine Hypothefe über die Natur des Stromes gegründet, und ohne eine grolse Wichtigkeit auf dielelbe zn legen, gebo ich Ge nur als ein, für den gegenwartigen Zuftand der WifTenfchaft hinreichendes, Mittel, Gach von der Erfcheinung Rechenfchaft zu geben.

Iclı nehme an, dafs der in einem Leiter erregte elektrifcize Strom nichts anderes $\mathrm{fey}$, als eine Folge von rafchen Zerletzungen und Wiederherftellungen der feinen Theilchen eigenthümlichen Elektricitut. Wie wird nun der elektriche Zuftand eines der Metalldrahtıe feyn, z. B. des am pofitiven Ende der Saule befeftigten? Um diefo zu erfahren, betrachton wir nur eine Reihe von Theilchen, da für alle übrigen daffelbe gilt. Das Theilchen $a$, welches unmittelbar mit der Flüfigkeit in Berührung fteht, ift + auf Seite der Flülifigkeit und - anf Seite des folgenden Theilcliens; das Theilchen $b$ if + gegen das - des Theilchens $a$, 


\section{[ 436 ]}

und - auf Seite des folgenden Theilchens $c$, und fo fort. Das + des Theilchens $a$ wird neutralifirt vom - des zerlézten flülfigen Theilchens, das + des Theilchens 6 vom - des Theilchens $a$ u. f. w. Ich nehme den Draht heraus; er befindet fich dann in einem $\mathrm{Zu}$ fiande, der, in Bezug auf die Elektricitat, demjenigen ziemlich ahnlich iff, welchen man, nach der Coulomb'fchen Theorie, im Stahle hinfichtlich des Magnetismus anninmt. Sein pofilives Ende ift nicht melir durch das negative Element der Flüfligkeit neutralifirt und kann ficls nicht vereinigen mit dem deflelben Theilchens $a$, welches von dem + des Theilchens $b$ zurückgehalten wird, wenigftens, wenn man, wie beim Magnetismus, eine Art von Coërcitivkraft annimmt. Der am negativen Ende der Süule befofig te Draht befindet ficls in einem ahnlichen $Z_{u}$ ftande; nur wird hier das - durch das + , und das + durch das - erfetzt.
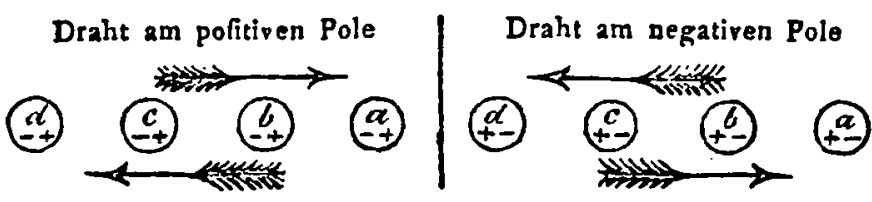

Verbinde ich die fo elektrifirten Drahte, entweder unter fich, oder mit cinem andern Leiter, mittelft des Galvanometers, fo wird jedes Theilchen fich wieder in feinen natïrlichen elektrilchen Zuftand verfezzen können, und fein + durch foin eignes - , und nicht durch das - des folgenden neutralifiren. Diefo Wiederherftellung wird zu eincm Strome von entgegengefetzter Richtinng Anla fs geben, wie der, welcher die Drălite elektrifirte. Denn diefer geht in jedem 


\section{[ 437 ]}

Theilchen rom - zum +, d. h. in der Richtung, in welcher die + fortgefülirt werden, wie es der obere Pfeil anzeigt; der andere, welcher die Viederherftellung bewirkt, geht ebenfalls in der Hiclitung, in der die + fortgeführt werden, wie es der untere Pfeil andeutet. Es ift daher nicht wunderbar, dafs der von den Drühten entwickelte Strom, dem Verfuche gemåls, in Richtung dem Strome entgegengefetzt ift, welcher ilınen von dem elektrodynamifchen Vermögen eingeprugt wird.

V Yarum ift aber ein flüfiger Leiter nöthig, um in den Drăhten, die die elektrodynamifche Eigenfchaft behalten haben, das Gleichgewicht wieder herzuftellen? - Wenn man gewifs leyn könnte, dals die Nothwendigkeit eines flüfligen Leiters nicht in der geringer Empfindlichkeit der Beobachtungsmittcl, fondern in der Natur der Erfclicinung ihren Grund laabe, lo glaube ich, kann man fie durch die folgende Bemerkung erklaren. Wenn die Leiter, welche zu zweien die vier Enden verbinden, beide metallifcher Art find, fo ift kein Grund vorhanden, weshalb das Gleichgewicht fich mehr in der einen Riclitung als in der andern wiederherftellen follte, walhrend, wenn einer der Leiter ein flüfiger ift, feine Gegenwart, indem fie die Kette fchliefat (eine nothwendige Bedingung zu der Wiederherftellung), nicht hindert, dafs der Strom mit grö̊serer Leichtigkeit durch den metallifchen Leiter gehe, und den Drähten erlaubt, fich zu entladen und auf iliren natürliclien Zufiand zurückzukommen.

Nach den obigen Betrachtungen würde alfo die natürliche Elektricität der Theilchen cines jeden Lei- 


\section{[ 430 ]}

ters in die beiden entgegengefetzten Theile zerfallen, und der + Theil immer nach der Richtung, welche der Strom befitzt, gewendet feyn. Eben fo, wie in den Strömen, würde es noch in jedem Leiter eine eigenthümliche Coërcitivkraft geben, die überwunden werden müfste, und daraus folgte, wie es auch dio Erfahrung beftatigt, dafs dor Dralit, wenn ex in den Strom geftellt wird, nicht augenblicklich in den elektrifclien $\mathrm{Zuftand}$ übergelien kann, und dafs, wenn er ilın einmal angenommen hat, er ihn auf kürzero oder langere Zeit behălt.

Wendet man auf diefe Hypothefe die Theorie der Magnete, in der Vorausfetzung von zwei magnetifchen Flülfigkeiten an, fo ift es leicht auf diefelbe Art zu erklarren, warum das ganze eine Ende pofitiv, das ganze andere negativift. Die Sătule und die beiden Drălite, worin fich diefelbe endigt, lafen fich als ein grofser Magnet betrachten, in welchem das magnetilche Fluicum durch das elektrifche erfetzt, fonft aber auf gleiche Weife angeordnet ift.

Die Annalıme einer, in ftarren Körpern dem Leitvermögen ungekelırt proportionalen, Coërcitivkraft für die Elektricitat lcheint mir übrigens viele andere elektrifche Erfcheinungen zu erklaren, wie ich in diefer Ablandlung, fo wie in andern Unterfuclungen über alnnliclie Gegenfiande, zu bemerken Gelegenheit haben werde.

Verfuche und Beobachtungen zur Slütze der obigen Theorie. - Die Analogie, welcho wir 2 wifchen einem Magneten und einen, der Wirkung des elektrilchen Stromes unterworfenen, Leiter aufgeffellt ha- 


\section{[ 439 ]}

ben, wird noclı rollkommner, wenn wir einen Platindraht betrachten, welcher zwei mit einem flüfigen Leiter gefüllte Schalchen verbindet, von denen jedes einen Pol der Săule aufrimmt. Man weifs, dafs dier fer Draht pofitiv ift an dem Ende, welches fich mit dem negativen Pole der Saule in einer Schale befindet, und negativ an dem andern Ende, welches mit dem pofitiven Pole derSaule in diefelbe Flüffigkeit getanclit jft. Diefer Draht, mit einem nicht elektrifirten Platindrahte an den Galvanoneter gebracht, wirkt wie ein Draht, welcher am + Pole der Sulu befeftigt war, oder wie ein an - Pole befindlich gewefener Draht, jo nachdem man das eine oder das andere feiner Enden in die Flülfgkeit taucht. Nicht blofs die bejden Enden des Drahtes, Condern jedes Stück von jlım, wirkt, und zwar wie das Ende, dem es zunáchft liegt. Nur if die Intenfitat der Wirkung um fo geringer, als es fich der Mitte năhert, wofelbft fie null ift; auch nimmt fie von den Enden aus folır rafch ab. Theilt man den numlichen Draht in zwei Theile und bringt beide an die Enden des Galvanometers, fo gefcliseht alles fo, als wenn man zwei getreunte Dralle, einen pofitiven und einen negativen, dalin verfelzt hälte. Die Riclıtung des Stromes ift in einem Falle wie im andern völlig dielelbe.

Ein wichtiger Verfuch, defTen Refultat fehr geeignet ift, die oben aufgeftellte Theorie zu beftatigen, befielit darin, dafs man den elektrodynamifch genachten Draht an irgend einer Stelle durchfchneidet und die Richtung des Stromes unterfucht, der entfteht, wenn man die beiden getrennten Stücke in denfelben Leiter taucht. Wie beim Magneten, mülten die ge- 


\section{[ 440 ]}

tronnten Stücke an den zuror vereinigt gowefenen Enden entgegengefetzte Pole erlualten. Die Richtung des Stromes zeigt, dafo diefes allerdingo der Fall ift, aber der Strom ift fchwach und oft null, wie diefs immcr gefchieht, wenn man fich nicht von den Drihten derjenigen Stücke bedient, welclıo in die, der Wirkung der Sulu ausgefelzte, Flüffigkeit eingetaucht waren. Sollte nicht die geringe Intenfitä des Stromes von der Schwache der Coërcitivkraft des Leiters in Bezıng auf die Elektricität herrühren? Diefer Gegenftand verdient weiter unterfucht zu werden. Gegenwartig bin ich mit einer Arbeit befchuftigt, in welcher ich fitclie, die Coërcitivkraft der ftarren Körper mit dem Loitvermögen derfelben zu vergleichen. Einige Verfuche fcheinen mir anzuzeigen, dafs diefe beiden Eigenfchaften, was ihre Intenfitat in jedem Körper betrift, fich umgekelırt zu einander verlıalten, das will fagen, dafs wenn man zwei Drăhte von gleichem Durchmeffer und gleicher Lange in diefelben Umftande verfetzt, derjenige, welcher der Cchlechtere Leiter ift, das gröfsere eloktrodynamifche Vermögen erhült. Indels, danit man diefem Refultate Zutrauen fclienken könne, mufs es erft durch mehr. abgeänderto und mit Sorgfalt angeftellte Verfuche erhalten worden feyn. Diefe find aber fehr mifslich, weil man den elektrifchen Zuftand des Drahts durch das Zerfchneiden deffelben und durch das Abwafchen und Abtrocknen feiner in die Flüßfigkeit getauchten Stücke leicht andern kann, und wenn man Letzteres unterlafst, können die an den Galvanometer gebrachten Stücke auf das Queckfilber, welches zur Verbindung dient, chemifch einwirken. 


\section{[ 441 ]}

Bevor ich fchliefse, wird es nicht überflüfig feyn, dafs ich bemerklich mache, welch ein grofser Unterfchied zwifchen dem Zuftande der gewöhnlichen elektrilchen Spannung und demjenigen elektrifchen $\mathrm{Zu}$ ftande herrfclit, in welchem fich die mit dem elektrodỷnamifchen Vermögen begabten Drăhte befinden.

2) Diefe Drahte zeigen niclit die mindefte Spur einer elektrifchen Spannung, felbft nicht mit den em$p_{\text {findlichften: Elektrofkopen. }}$

2) Die Berührung mit den beften Leitern, das Reiben und ander mechanifolic Operationen entziehen ihnen niclit das elektro-dynamifche Vermögen.

3) Der elektrilcho Zuftand, in welchen fich der Draht verfetzt, der die beiden Schulchen, in denen die Zerfetzung gefchieht, verbindet, zeigt keine Aehnlichkeit mit dem elektrilchen Zuftando des Bandes von Volta; denn er (der Draht) giebt keine Anzeigen von elektrifcher Spannung. Ich habe niemals mit einem flülfigen Leiter eine Wirkung, der ahnlich, wolcle der Dralit giebt, hervorbringen können. So wurde ein reclangulares Gefufs, anf deften Boden fich ein flülliger Leiter in einer. Schicht von der Dicke eines halben Lolles befand, nachdem es eine Zoit lang in dum Kreile der Süule gewelen war, mit dem Galvanor:teter in Berülırung gefetzt, unid zwar durch zwei l'latinfpilzen, die man forgfaltig genau da eintauchte, wo vorhin die Pole der Saule befindlich wasen; und niemals trat der kleinfte Strom oin.

4) Wenn endlich der elektrifche Zuftand des Drahts davon herrührte, dafs die Elektricitat, wenn lie eine fohlechtleitende Flüligkeit trift, nicht hinAunal, d. Pbysik. B.86. St. 3. J.1827. St.7. 


\section{$\left[\begin{array}{lll}4.12 & 1\end{array}\right.$}

durch könnte und folglich in dem Dralıte angeliduft bliebe, fo muiliste das elektro - dynamifclie, Vermögeu "1m to intculiver leyn', als die Fluiligkeit ein Schlechwerer Leiter wate. Allein gerade das Gegentheil findet Stalt.

Beınerken wir überdiefs, dafs der Lejter ein defto kräftigeres Vermögen erlangt, je dicker er $i f t$, und $j$ t melır Berïhrungspunhte er der Flitfigkeit darbietel. Mau kanu felbft mit drei llatinplatlen, die durch oinen nïlrigen Leiter getrennt find, die elektro-dynanifilie Kraft in deni Grade condeufiren, dals nan, naclidem diefe l'latten einige Zeit in Kreili der Voltalchen Sulule verweilt haben, einen Strom erlialt, der der $\mathrm{Na}$ del eine auf melirere Augenblicke conftante Ablenkung voll $20^{\circ}$ ertheilt. Merkw ürdig ift es aber, dals, nachdem man alle, walirend der Volifafchen Aclion zwifchen den Platiuplatton befindliclıo Flülfigkeit fortgenonmen liat, und eine andere an ihrer Stelle nimnt, die elektro-dynamifclie Wirkung in gleicher Weife Statt findet. Dieler letzte Verliuch beweift, wie die vorhergelienden, dafs es keinesweges eine gegenfeitige Reaction zwifthen der Flïlfigkeit und dem, nut dieler zuliumen in der Kette befindliclien, Leiter ift, fondern, dal's ein eigenthümlichor Zuftand, in welchen fich blol's der Leiter verletzt, die Erlcheinnng bewirkt, deren Unterfucliung der' Zweck diefer Ablıandlung gewelen ifi. 


\section{[ 443$]$}

R 1 : D I 1 : k

Aus dem Vorhergehenden glaube ich fchliefsen zn können: 1) dafs die ftarren Körper, wenn fie als Leiter der Elektricitat gedient haben und dann unter die günftigen Umftände gebraclit werden, die Eigenfchift erlaugen, dafs fie zul einem Strame Anlafs geben, eine Eigenlachaft, die man das elektro-dynamifoke Vermögen nennen kann. - 2) Dafs diefe Leiter nur dann diefes Vermồgen erlangen und entwickeln können, wenn ein Theil der Kette einen flilfigen, nicht metallifclien Leiter einfchliefst. - 3) Dafs dio flülfigen Jeiter, 'nnter dielélben Umftrinde verfetzi, nicht fahing find, wie die ftarren, diefo Eigenlchaft zu erlangen. - 4) Dafs alle Uniftande, welche die Entftehung der Erfcheinung begleiten, zu der Folgerung zu fäitren fclieinen, dals der Stronı durch eine folgweife Zerletzing und Viederherftellung der naturliclien Elektricitat der einzelnen Theilchen entfieht, und dal's es in den ftarren Leitern eine Coërcitivkraft giebt, vermöge welcher fie pirı grölsere oder gerin-. sere Zeit hindurch in dem elektrifthen Zuftande, welchen ihnen der Durcligang des Stroms eingepragt hat, beharren können. 